\title{
Biexciton Binding Energy and Line width of Single Quantum Dots at Room Temperature
}

\author{
Sander J. W. Vonk, Bart A. J. Heemskerk, Robert C. Keitel, Stijn O. M. Hinterding, Jaco J. Geuchies,
} Arjan J. Houtepen, and Freddy T. Rabouw*

Cite This: Nano Lett. 2021, 21, 5760-5766

Read Online

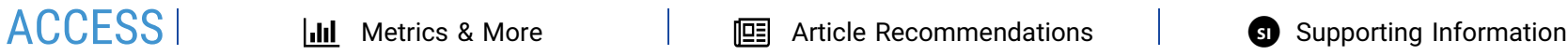

ABSTRACT: Broadening of multiexciton emission from colloidal quantum dots (QDs) at room temperature is important for their use in high-power applications, but an in-depth characterization has not been possible until now. We present and apply a novel spectroscopic method to quantify the biexciton line width and biexciton binding energy of single $\mathrm{CdSe} / \mathrm{CdS} / \mathrm{ZnS}$ colloidal QDs at room temperature. In our method, which we term "cascade spectroscopy", we select emission events from the biexciton cascade and reconstruct their spectrum. The biexciton has an average emission line width of $86 \mathrm{meV}$ on the single-QD scale, similar to that of the exciton. Variations in the biexciton repulsion $\left(E_{\mathrm{b}}=4.0 \pm 3.1 \mathrm{meV}\right.$; mean \pm standard deviation of 15 QDs) are correlated with but are more narrowly distributed than variations in the exciton energy $(10.0 \mathrm{meV}$

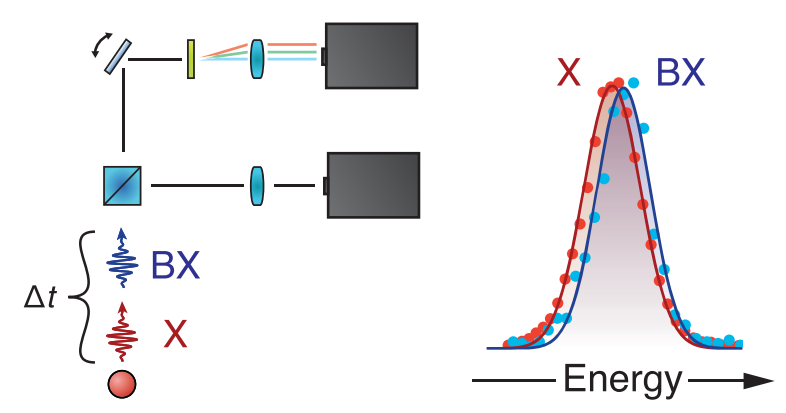
standard deviation). Using a simple quantum-mechanical model, we conclude that inhomogeneous broadening in our sample is primarily due to variations in the CdS shell thickness.

KEYWORDS: quantum dots, single-quantum-dot spectroscopy, multiexciton emission, biexciton line width

ince the first report of colloidal quantum-dot (QD) $\checkmark$ synthesis 30 years ago, ${ }^{1}$ significant research effort has been devoted to reaching high emission efficiencies and pure emission colors tunable over the entire visible range. However, variations in particle size, which reduce the color purity because of inhomogeneous broadening, cannot be entirely avoided. Inhomogeneous and homogeneous broadening ${ }^{2-6}$ of QD emission at room temperature, directly relevant for applications in displays and lamps, has been studied extensively on the ensemble and single-QD level. Previous studies have focused on QD emission in the regime of weak optical excitation, where QD fluorescence originates from the singleexciton excited state. Prospective new applications-such as lasing and high-power lighting-inherently demand stronger excitation, which creates multiexciton states with optical properties different from those of single excitons, ${ }^{7,8}$ most notably a lower emission efficiency because of Auger recombination. ${ }^{9,10}$

The spectral characteristics of multiexcitons have remained largely unexplored. Studying multiexciton states is difficult because, under strong excitation, the simultaneous presence of trions, biexcitons, and higher multiexcitons obscures the investigation of any particular multiexciton state. Only at cryogenic temperatures can multi- and single-exciton emission be separated on the basis of polarization ${ }^{11,12}$ or by spectral selection. ${ }^{13}$ At room temperature, such selections are difficult because the energy splitting between fine-structure states and the spectral shifts between single excitons and multiexcitons are small (a few $\mathrm{meV}$ ) in comparison to the emission line widths (tens of $\mathrm{meV}$ ). Photon-correlation investigations of multiexciton $^{14-22}$ emission efficiencies at room temperature have revealed enormous heterogeneities with no apparent link to the single-exciton properties. ${ }^{20,23-26}$ A detailed characterization of multiexciton emission energies and the variations thereof at the single-QD level is important for high-power applications $^{27-29}$ but has not yet been achieved.

In this Letter, we measure the biexciton spectrum of single QDs at room temperature. We employ a novel spectroscopic technique, which we call "cascade spectroscopy". In this technique, we identify biexciton cascades from the emission of a single $\mathrm{QD}$ by cross-correlating the signal recorded by two time-resolved single-photon detectors. On one of the two detectors we achieve energy resolution, which allows us to reconstruct the biexciton emission spectra. We observe a repulsive biexciton of single $\mathrm{CdSe} / \mathrm{CdS} / \mathrm{ZnS}$ core-shell-shell

Received: April 20, 2021

Revised: June 12, 2021

Published: June 16, 2021 

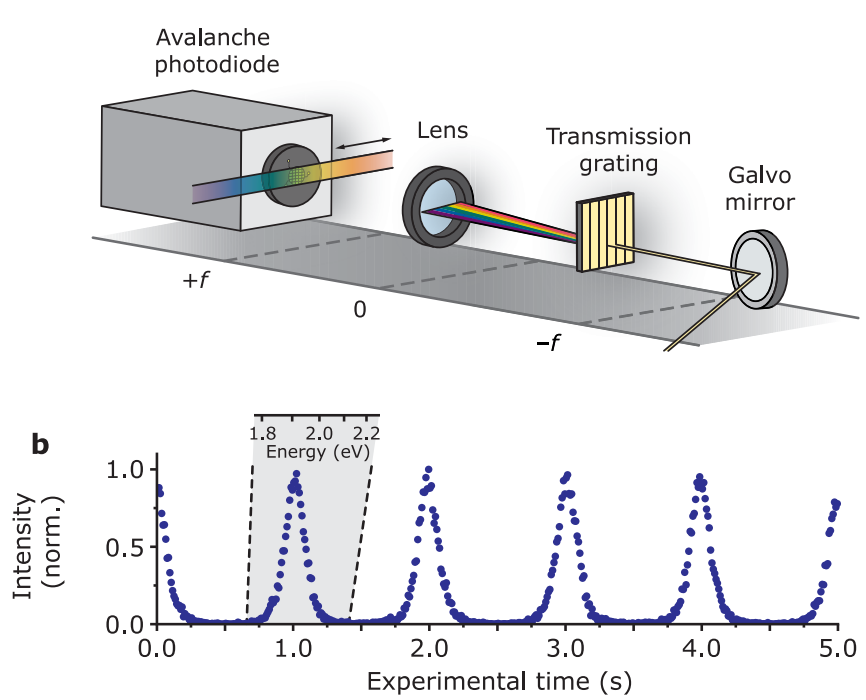
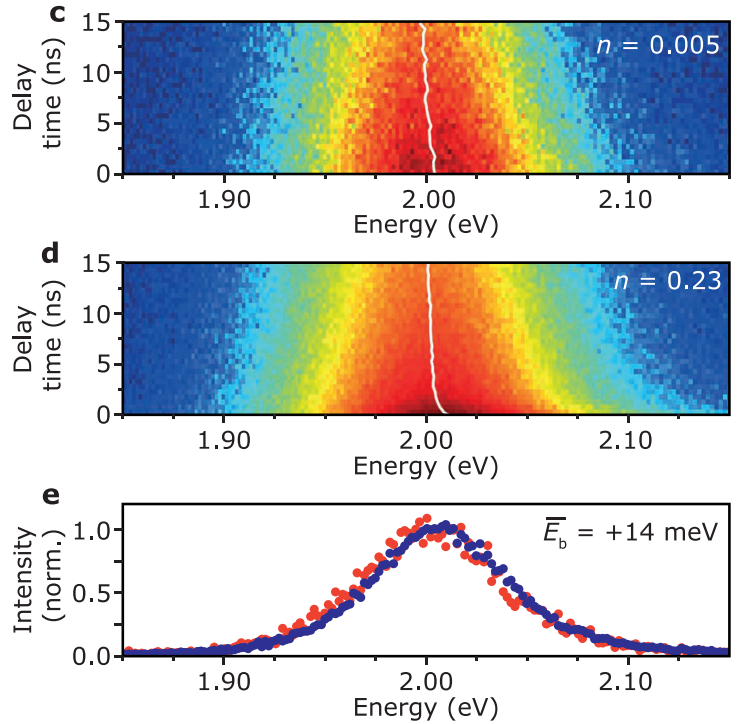

Figure 1. (a) Schematic of the galvo-APD setup. The QD emission is directed to a periodically rotating galvo mirror, which changes the angle of incidence on a transmission grating. Fourier imaging of the grating plane projects part of the QD emission spectrum onto an avalanche photodiode (APD). (b) Periodically rotating the galvo mirror with $0.5 \mathrm{~Hz}$ scans the emission spectrum of the QDs (pulsed excitation $2.5 \mathrm{MHz}$ ) over the APD twice every $2 \mathrm{~s}$. We can convert the experimental time $T$ to a photon energy (see inset). (c, d) Time-resolved emission spectra of a micrometerthick layer of QDs on glass at increasing excitation-pulse energy. The white lines indicate the emission maximum as a function of delay time. At the highest power, the spectrum is slightly blue shifted at delay times of $0-2 \mathrm{~ns}$ because of the contribution of repulsive biexcitons. (e) Emission spectrum integrated between 0 and $0.6 \mathrm{~ns}$ delay times for $n=0.005$ (red, panel c) and $n=0.23$ (blue, panel d) excitons per pulse. By globally fitting a series of spectra for different fluences, we obtain an ensemble biexciton shift of $\bar{E}_{\mathrm{b}}=+14 \mathrm{meV}$.

QDs, commonly used for lasing, ${ }^{27}$ consistent with powerdependent measurements on the ensemble scale and with previous studies of thick-shelled core-shell QDs. ${ }^{30}$ The singleQD biexciton emission line width is $86 \mathrm{meV}$ on average (full width at half-maximum; fwhm). This matches the line width of the single-QD exciton emission of our QDs and those of other groups. ${ }^{3,28}$ We find single-QD biexciton shifts of $E_{\mathrm{b}}=4.0 \pm 3.1$ $\mathrm{meV}$, which are slightly negatively correlated with but more narrowly distributed than the single- $Q D$ exciton energies (variation of $10.0 \mathrm{meV}$ ). Comparing these correlations and variations to simple quantum-mechanical calculations of multicarrier states in QDs, we conclude that both are predominantly due to variations in the thickness of the CdS shell. We thus present the first method to characterize singleQD biexciton spectra at room temperature, which will guide the development of synthesis strategies aimed at minimizing inhomogeneous broadening of biexciton emission for highpower applications.

We first characterize the biexciton emission of our CdSe/ $\mathrm{CdS} / \mathrm{ZnS}$ core-shell-shell sample (section $\mathrm{S} 1$ in the Supporting Information) on the ensemble scale, as a micrometer-thick layer on a cover slip, using energy-resolved time-correlated single-photon counting. Figure 1a shows the setup, which we term the "galvo-APD" setup. A galvo mirror periodically varies the incidence angle of the $\mathrm{QD}$ emission onto a transmissive diffraction grating (300 lines $/ \mathrm{mm}$ ). Fourier imaging of the grating plane projects part of the QD spectrum onto an avalanche photodiode (APD) with a resolution $\Delta \lambda \approx$ $a L / f$, where $a$ is the detector size $(20 \mu \mathrm{m}), L$ is the groove spacing $(3.3 \mu \mathrm{m})$, and $f$ is the focal distance $(20 \mathrm{~mm})$. These optical elements give a resolution of approximately $\Delta \lambda=3 \mathrm{~nm}$ (section S2.1 in the Supporting Information). Periodic scanning (Figure $1 \mathrm{~b}, 0.5 \mathrm{~Hz}$ ) measures the emission spectrum twice every $2 \mathrm{~s}$. We perform a pulsed-excitation experiment (repetition rate $2.5 \mathrm{MHz}$ ), where we deduce the energy of each detected photon from the detection time $T$ since the beginning of the experiment (see inset of Figure 1b). For each photon, we simultaneously measure the delay time $t$ with respect to the previous laser pulse.

Figure 1c,d shows time-resolved emission maps, i.e. emission spectrum versus delay time, at different excitation powers, measured with our galvo-APD setup. At the lowest power (estimated number of excitons per QD per pulse $n=0.005$, Figure 1c) the emission peaks at $2.002 \mathrm{eV}$ on the interval $t=$ $0-0.6 \mathrm{~ns}$. It then slowly shifts to lower energies over the next $15 \mathrm{~ns}$, likely due to inhomogeneous broadening of the emission $^{31}$ and exciton diffusion. ${ }^{32}$ At higher powers $(n=$ 0.23 , Figure $1 \mathrm{~d}$ ) an additional slightly bluer contribution to the emission appears; the emission peaks at $2.007 \mathrm{eV}$ on the interval $t=0-0.6 \mathrm{~ns}$. Figure 1e shows the integrated emission spectra for $t=0-0.6 \mathrm{~ns}$ for the low fluence (red) and the high fluence (blue). This indicates a repulsive biexciton; i.e., the ensemble-scale biexciton shift is positive, $\bar{E}_{\mathrm{b}}=\bar{E}_{\mathrm{BX}}-\bar{E}_{\mathrm{X}}>0$, where $\bar{E}_{\mathrm{BX}}$ and $\bar{E}_{\mathrm{X}}$ are the ensemble-scale biexciton and exciton emission energies, respectively. We expect negligible contribution of triexcitons at the excitation fluences used. Nevertheless, extracting a quantitative value for $\bar{E}_{\mathrm{b}}$ remains difficult, because the majority of the signal originates from the exciton and the shift between exciton and biexciton emission is much smaller than the line width due to inhomogeneous and homogeneous broadening. A global-fit procedure (section S3 in the Supporting Information) yields a best estimate for the ensemble-scale biexciton shift of $\bar{E}_{\mathrm{b}}=14 \mathrm{meV}$.

Experiments on single QDs allow us to unambiguously distinguish between biexciton and exciton photons and to directly extract the biexciton shift and the biexciton emission spectrum. We split the emission of a single QD using a Hanbury-Brown-Twiss (HBT) setup (Figure 2a), in which one of the detection channels uses a regular APD while the 
a

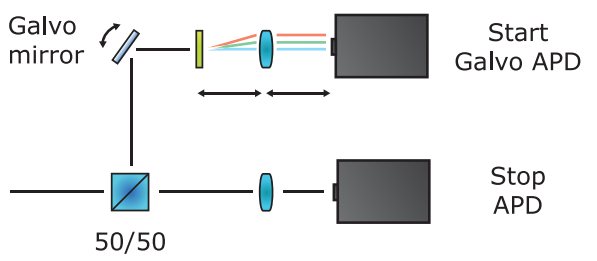

b

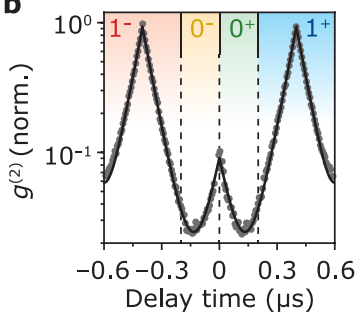

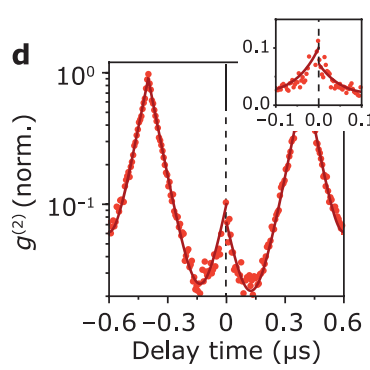

c
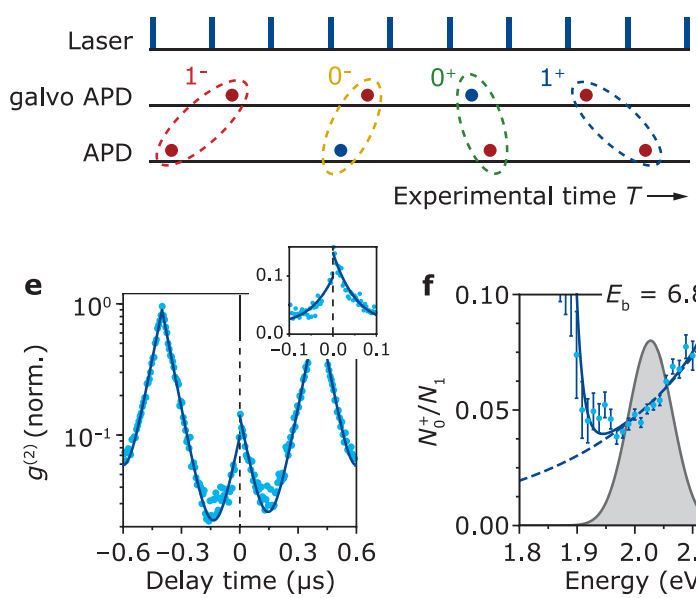

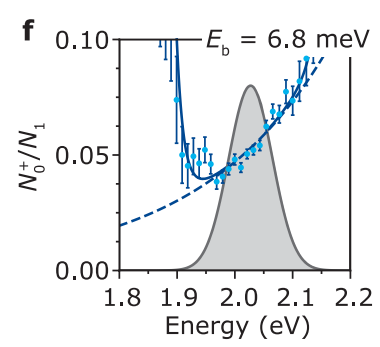

Figure 2. (a) Schematic of the cascade spectroscopy setup. The emission of a single QD is split equally over the energy-resolved "galvo APD" (Figure 1a) and a regular APD. (b) Photon correlation function $g^{(2)}$ of a single QD (repetition rate $2.5 \mathrm{MHz}$, estimated average number of excitons per pulse $\mu=0.1$ ) integrated over the entire experiment, showing a biexciton efficiency of $\eta_{\mathrm{BX}}=10.5 \%$. The highlights mark the delay time ranges where events of types $1^{-}, 0^{-}, 0^{+}$, and $1^{+}$contribute. (c) Overview of possible ordered-emission events. The laser pulses are the vertical blue lines. Events wherein two photons (dots) are detected following two consecutive laser pulses are highlighted $1^{+}$and $1^{-}$, depending on the order of the detector clicks. Two photons detected after a single laser pulse, indicating biexciton cascade emission, are highlighted $0^{+}$and $0^{-}$. The first photon of a cascade is a biexciton photon (blue dots); all other photons most likely originate from the single-exciton state (red dots). (d, e) Energy-resolved $g^{(2)}\left(\log y\right.$-scale) for low (d) and high (e) galvo-APD detection energies. Lower galvo-APD detection energies $E_{\operatorname{det}}=1.95-1.99 \mathrm{eV}$ yield a ratio $N_{0}^{+} /$ $N_{0}^{-}<1$, while for blue-shifted detection energies $E_{\text {det }}=2.08-2.16 \mathrm{eV}, N_{0}^{+} / N_{0}^{-}>1$. This indicates a repulsive biexciton. The insets (linear $y$ scale) are enlarged views to show $N_{0}^{+} / N_{0}^{-}$more clearly. We discarded afterglow events from panels b, d, and e (Figure S3 in the Supporting Information). (f) $N_{0}^{+} / N_{1}$ as a function of galvo-APD detection energy $E_{\text {det }}$. The gray spectrum shows the exciton emission of this single QD. The error bars are calculated assuming shot noise. By fitting eq 2 to the data, we extract a single-QD biexciton shift of $E_{\mathrm{b}}=6.8 \pm 1.0 \mathrm{meV}$.

other uses the galvo-APD setup (70 lines $/ \mathrm{mm}, \Delta \lambda=13 \mathrm{~nm}$ ), introduced in Figure 1a.

From the energy-resolved measurement, we can reconstruct the same photon-correlation function $g^{(2)}$ as obtained with a conventional HBT setup, by simply integrating $g^{(2)}$ over the total experimental time of $30-60 \mathrm{~min}$ (section S4.2 in the Supporting Information). Figure $2 \mathrm{~b}$ shows the result for $\mathrm{a}$ typical single QD from our batch. We performed a blind-time analysis to confirm that the zero-delay peak is due to biexcitoncascade events, while the side peaks are due to exciton-exciton pairs following consecutive laser pulses (Figure S4 in the Supporting Information). ${ }^{33}$ The correlation function measured in the limit of weak excitation reveals the biexciton-to-exciton efficiency ratio $\eta_{\mathrm{BX}} / \eta_{\mathrm{X}}$ from the number of photon-pair events in the zero-delay peak $N_{0}$ and the number of photon-pair pairs in a side peak $N_{1}:{ }^{14}$

$$
\frac{N_{0}}{N_{1}}=\frac{\eta_{\mathrm{BX}}}{\eta_{\mathrm{X}}}
$$

We find a biexciton efficiency of $\eta_{\mathrm{BX}}=10.5 \%$ (assuming $\eta_{\mathrm{X}}=$ $\left.1^{14}\right)$, which is a typical value for thick-shell CdSe/CdS/ZnS core-shell-shell QDs. ${ }^{14,26}$

In our correlation analysis, the galvo APD acts as the "start" channel and the regular APD as the "stop" channel. In our definition of $g^{(2)}$, the biexciton cascade emission wherein the first photon arrived at the galvo APD thus appears in the zerodelay peak at positive delay times (highlighted with $0^{+}$in Figure $2 \mathrm{~b}, \mathrm{c})$; cascade emission wherein the first photon arrived at the regular APD appears in the zero-delay peak at negative delay times (highlighted with $0^{-}$in Figure $2 \mathrm{~b}, \mathrm{c}$ ). Similarly, for the side peaks, the order of the detector clicks determines whether an exciton-exciton emission pair appears at positive or negative delay times (compare $1^{-}$and $1^{+}$in Figure $2 \mathrm{~b}, \mathrm{c}$ ).

The relative areas of the peaks in the correlation function $g^{(2)}$ for a specific galvo-APD detection energy $E_{\text {det }}$ contain information about the biexciton emission spectrum. Indeed, the number of recorded photon pairs of types $1^{-}, 0^{-}, 0^{+}$, and $1^{+}$ depend on $E_{\text {det }}$ and on the emission spectra of biexcitons and excitons. Successful detection of photon pairs of type $1^{-}, 0^{-}$, and $1^{+}$require the galvo APD to record an exciton photon (section S4.3 in the Supporting Information); thus, the number of detection events is proportional to the brightness of the exciton at $E_{\text {det }}$. In contrast, for events of type $0^{+}$the galvo APD must record a biexciton photon; thus, the number of such events is proportional to the biexciton brightness at $E_{\operatorname{det}}$. A comparison of $g^{(2)}$ for $E_{\text {det }}=1.95-1.99 \mathrm{eV}$ (Figure 2d) and for $E_{\text {det }}=2.08-2.16 \mathrm{eV}$ (Figure 2e) shows the consequence: the total number of events of types $0^{-}$and $0^{+}$, which we denote $N_{0}^{-}$ and $N_{0}^{+}$, are unequal and dependent on $E_{\text {det }}$. More precisely, we observe that $N_{0}^{-}>N_{0}^{+}$in Figure $2 \mathrm{~d}$ and $N_{0}^{-}<N_{0}^{+}$in Figure 2e (highlighted in the insets). Hence, a low $E_{\text {det }}$ favors the recording of exciton photons on the galvo APD, while a high $E_{\text {det }}$ favors the recording of biexciton photons. This proves that this single $\mathrm{QD}$ has a repulsive biexciton: i.e., the biexciton emission energy $E_{\mathrm{BX}}$ is higher than the exciton emission energy $E_{\mathrm{X}}$. With a quantitative analysis, we can determine the biexciton shift $E_{\mathrm{b}}=E_{\mathrm{BX}}-E_{\mathrm{X}}$ on the single-QD level. We compare the number of events recorded of type $0^{+}$(positivetime area of the zero-delay peak $N_{0}^{+}$) to the number of events recorded of types $1^{-}$and $1^{+}$(side-peak areas $N_{1}^{-}$and $N_{1}^{+}$). To improve the signal-to-noise ratio, we take the average $N_{1}=N_{1}^{-}$ $+N_{1}^{+}$. Realizing that $N_{0}^{+}$scales with the biexciton emission spectrum $I_{\mathrm{BX}}(E)$ peaking at $E_{\mathrm{BX}}=E_{\mathrm{X}}+E_{\mathrm{b}}$ and $N_{1}$ with the 

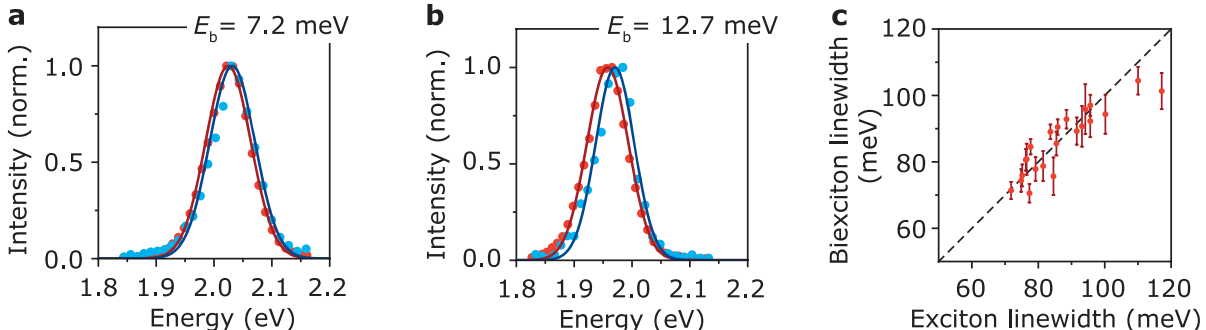

Figure 3. (a) Biexciton (blue) and exciton (red) emission spectra of the single QD analyzed in Figure 2. The biexciton spectrum was extracted from the positive-time area $N_{0}^{+}$of the zero-delay peak in the energy-resolved photon-correlation function. We measure a biexciton shift of $E_{\mathrm{b}}=7.2$ $\pm 2.1 \mathrm{meV}$. (b) A different QD with exciton with a larger biexciton shift of $E_{\mathrm{b}}=12.7 \pm 1.9 \mathrm{meV}$. (c) Biexciton and exciton line widths (fwhm) of 22 single QDs measured. Error bars are standard errors of the Gaussian fit.

exciton emission spectrum $I_{\mathrm{X}}(E)$ peaking at $E_{\mathrm{X}}$, we can derive (section S4.1 in the Supporting Information)

$$
\frac{N_{0}^{+}}{N_{1}}=\frac{1}{2} \frac{\eta_{\mathrm{BX}}}{\eta_{\mathrm{X}}} \frac{I_{\mathrm{BX}}\left(E_{\mathrm{det}}\right)}{I_{\mathrm{X}}\left(E_{\mathrm{det}}\right)}
$$

We observe that $N_{0}^{+} / N_{1}$ increases as the galvo-APD detection energy increases for $E_{\mathrm{det}}=1.97-2.07 \mathrm{eV}$ (Figure $2 \mathrm{e})$. Outside this range of $E_{\mathrm{det}}$, the detection efficiency of both biexciton and exciton emission is low and dark counts affect the measurement. We fit $N_{0}^{+} / N_{1}$ as a function of detection energy $E_{\text {det }}$ using eq 2 (dashed blue line), fixing the previously determined biexciton efficiency, assuming Gaussian exciton and biexciton spectra with equal line width (fwhm $=85 \mathrm{meV}$, gray exciton spectrum inset) and using the biexciton shift $E_{\mathrm{b}}$ as the only fit parameter. The solid blue line is calculated including the contribution of dark counts (section S4.3 in the Supporting Information), which yields a match with the data over the full range of $E_{\text {det }}$. We obtain a biexciton shift $E_{\mathrm{b}}=6.8$ $\pm 1.0 \mathrm{meV}$ for this single QD.

Understanding the different cascade events that occur in our experiment, we can now reconstruct the single-QD biexciton spectrum directly from the positive-time area $N_{0}^{+}$of the zerodelay peak. The absolute value of $N_{0}^{+}$as a function of $E_{\text {det }}$ is proportional to the biexciton emission spectrum $I_{\mathrm{BX}}$. Similarly, we obtain the exciton emission spectrum $I_{\mathrm{X}}$ from $N_{1}$ as a function of $E_{\text {det }}$. The contribution of photon pairs to $N_{0}^{+}$and $N_{1}$ originating from a transient low-efficiency gray state due to blinking is negligible (Figure S6 in the Supporting Information), since the count rate of photon pairs scales quadratically with emission efficiency. Figure 3a shows the biexciton and exciton emission spectra obtained with this analysis procedure of the same $\mathrm{QD}$ as described in Figure 2. The exciton spectrum (red dots) has a maximum at $E_{\mathrm{X}}=2.025$ $\mathrm{eV}$ and a fwhm of $92 \mathrm{meV}$. The emission is broadened by spectral diffusion and slightly by the finite resolution of the setup (Figure S2 in the Supporting Information). Interestingly, we measure a biexciton spectrum (blue dots) with an equal fwhm of $89 \mathrm{meV}$, which confirms the assumption of equal exciton and biexciton line widths that we used in fitting eq 2 to the data in Figure 2e. From the difference in fitted exciton and biexciton emission maxima we obtain a biexciton shift of $E_{\mathrm{b}}=$ $7.2 \pm 2.1 \mathrm{meV}$, very similar to the value found from the fit in Figure 2e.

Figure $3 \mathrm{~b}$ shows the emission spectra of a different QD from the synthesis batch. The exciton emission peaks at $E_{\mathrm{X}}=1.958$ $\mathrm{eV}$, and interestingly, the biexciton is significantly more repulsive: $E_{\mathrm{b}}=12.7 \pm 1.9 \mathrm{meV}$. Again, we observe equal line widths of the exciton and biexciton emission of $85 \mathrm{meV}$. Figure
$3 c$ shows the biexciton and exciton emission line widths obtained from 22 single-QD measurements (Figures S7-S9 in the Supporting Information). The single-QD line widths of biexciton and exciton emission are distributed between 70 and $100 \mathrm{meV}$ fwhm, with a mean of 86 and $87 \mathrm{meV}$, respectively, and are strongly correlated; the biexciton and emission line widths of a single QD typically differ by no more than $10 \mathrm{meV}$ fwhm. Equal line widths for the biexciton and exciton were reported before for epitaxially grown QDs over a wide temperature range from cryogenic temperatures up to room temperature. ${ }^{34}$ Our experimental finding shows that the homogeneous emission broadening (determined by a combination of spectral diffusion, phonon coupling, and fine structure $^{3,34-36}$ ) of different single QDs varies, but it affects the exciton and biexciton emission line widths by the same amount.

Figure $4 \mathrm{a}$ shows an overview of the biexciton shifts and exciton energies found. Of the 22 single-QD measurements, 15 were randomly selected QDs (blue dots in Figure 4a) and 7 QDs were specially selected for their low-energy emission (red dots in Figure 4a). We find that the variations in the exciton emission maximum ( $10.0 \mathrm{meV}$ standard deviation) exceed the variations in the biexciton shift $(3.1 \mathrm{meV})$ by almost a factor of 4. Additionally, we observe on average a slightly more repulsive biexciton $E_{\mathrm{b}}=+9.4 \mathrm{meV}$ for the selected $\mathrm{QDs}$ in comparison to a weakly repulsive biexciton $E_{\mathrm{b}}=+4.0 \mathrm{meV}$ for randomly selected QDs.

To understand the variations in exciton energy and biexciton shift, we set up a simple quantum-mechanical effective-mass model of multicarrier states in a spherical core-shell-shell QD. Coulomb interactions between carriers are included as a perturbation to the particle-in-a-box confinement energies (section S5 in the Supporting Information). We compute the biexciton shift $E_{\mathrm{b}}$ and exciton energy $E_{\mathrm{X}}$ of core-shell geometries with different $\mathrm{CdSe}$ core radii $a$ and $\mathrm{CdSe} / \mathrm{CdS}$ core-shell radii $b$. Figure $4 \mathrm{~b}$ shows the results of the calculations in a way that facilitates comparison to the experimental data of Figure 4a: a grid of contour lines lays out which QD geometry $(a, b)$ produces which values of $E_{\mathrm{b}}$ and $E_{X}$.

We can determine the dominant geometrical contribution to inhomogeneous emission broadening in our QD sample by comparing the calculations (grid lines in Figure $4 \mathrm{~b}$ ) to the experimental distribution of QD properties (highlighted by the gray area). The experimental variation in $E_{X}$ exceeds the variation in $E_{\mathrm{b}}$ by almost a factor of 4 , and they are slightly negatively correlated. This is consistent with energy variations due to a distribution of core-shell radii $b$, while a distribution 

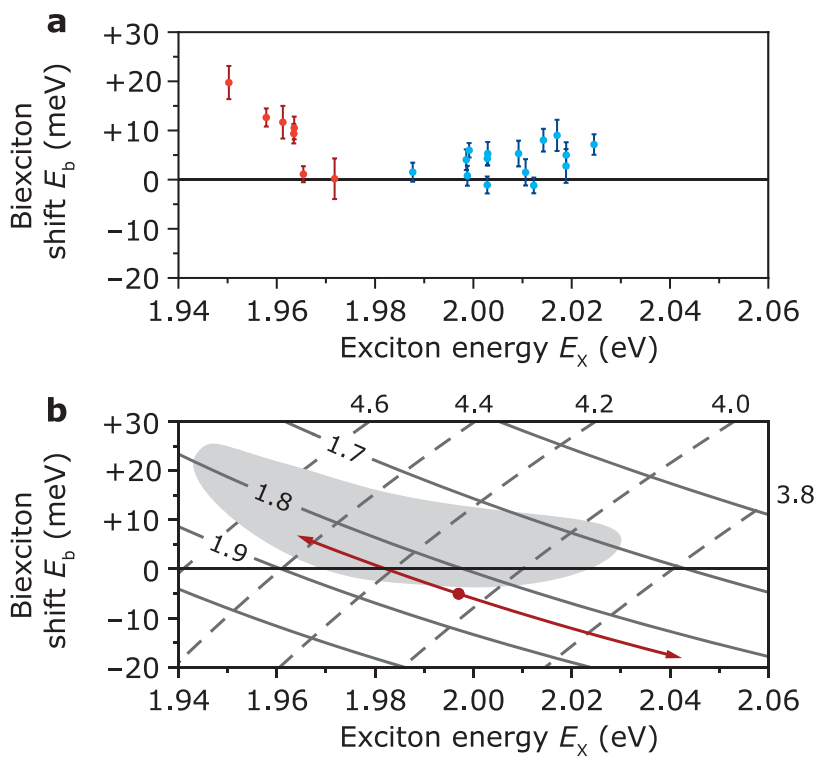

Figure 4. (a) Biexciton shift $E_{\mathrm{b}}$ versus exciton energy $E_{\mathrm{X}}$ for randomly selected QDs (blue) and for QDs selected for their low-energy exciton emission (red). Error bars denote one standard error of the fit. We observe a slight negative correlation between biexciton shift and exciton energy. (b) Dependence of $E_{\mathrm{b}}$ and $E_{\mathrm{X}}$ on CdSe core radius a and $\mathrm{CdSe} / \mathrm{CdS}$ core-inner-shell radius $b$, calculated with an effectivemass model that includes Coulomb interactions between carriers as a perturbation to second order (section S5 in the Supporting Information). The QD geometry is given as grid lines for $a$ (core radius, solid lines) and $b$ (core-inner-shell radius, dashed lines). The gray-shaded area highlights the range of exciton energies and biexciton shifts found experimentally. The red point and arrows are the calculated exciton energy and biexciton shift for the independently determined values of $a=1.84 \mathrm{~nm}$ (from the absorption spectrum of the cores) and $b=4.1 \pm 0.4 \mathrm{~nm}$ (from electron microscopy).

of core radii $a$ appears to be of lesser importance. Indeed, the solid contour lines in Figure $4 b$, running along directions of constant $a$, slope in approximately the same direction as the experimental distribution of single-QD emission energies. The calculations would predict that our experimental finding of the biexciton and exciton energies are due to a distribution of QD geometries with $a=1.82 \pm 0.05 \mathrm{~nm}$ and $b=4.3 \pm 0.3 \mathrm{~nm}$. These calculated values match well with the values of $a=1.84$ $\mathrm{nm}$ determined from the absorption spectrum of the cores and $b=4.1 \pm 0.4 \mathrm{~nm}$ (mean \pm standard deviation) from electron microscopy (red arrows in Figure 4b). A dominant contribution of variations in the CdSe/CdS core-shell size $b$ to inhomogeneous broadening could be expected on the basis of our synthesis procedure and those of many others. ${ }^{28,37,38}$ For the core synthesis, growth is fast and is stopped before size defocusing of $a$ sets in. ${ }^{39}$ Shells, on the other hand, are grown at a low growth rate to avoid secondary nucleation, which may lead to Ostwald ripening effects that widen the distribution of shell thicknesses. ${ }^{40}$

Using our effective-mass model, we can intuitively understand why variations in core-shell size $b$ produce the observed distribution of biexciton and exciton energies. When Coulomb interactions are included as a first-order perturbation, the exciton energy is

$$
E_{\mathrm{X}}^{(1)}=E_{\text {conf }, \mathrm{e}}+E_{\text {conf }, \mathrm{h}}+J_{\mathrm{eh}}
$$

where $E_{\text {conf, e }}$ and $E_{\text {conf, h }}$ are the particle-in-a-box confinement energies of the single-electron (e) and single-hole (h) $1 \mathrm{~S}$ states, respectively, and $J_{i j}$ is the Coulomb interaction between carriers $i$ and $j$. Similarly, the biexciton shift including Coulomb interactions to first order is

$$
E_{\mathrm{b}}^{(1)}=2 J_{\mathrm{eh}}+J_{\mathrm{hh}}+J_{\mathrm{ee}}
$$

This description yields analytical expressions for the dependence of $E_{\mathrm{X}}^{(1)}$ and $E_{\mathrm{b}}^{(1)}$ on $a$ and $b$ in the limit that $b$ $\gg a$ (section S5.1 in the Supporting Information). As inhomogeneous broadening in our QD batch appears mostly due to variations in $b$, we can understand it from the derivatives of eqs 3 and 4 with respect to $b$

$$
\begin{aligned}
& \frac{\partial E_{\mathrm{X}}^{(1)}}{\partial b}=\frac{-\hbar^{2} \pi^{2}}{m_{\mathrm{e}} b^{3}}+2.44 \frac{e^{2}}{4 \pi \varepsilon \varepsilon_{0} b^{2}} \\
& \frac{\partial E_{\mathrm{b}}^{(1)}}{\partial b}=3.09 \frac{e^{2}}{4 \pi \varepsilon \varepsilon_{0} b^{2}}
\end{aligned}
$$

where $\hbar$ is the reduced Planck's constant, $m_{\mathrm{e}}$ the effective mass of the electron, $e$ the elementary charge, $\varepsilon_{0}$ the permittivity of the vacuum, and $\varepsilon$ the relative permittivity of the QD material. Evaluating eqs 5 and 6 at a core-shell radius of $b=4.1 \mathrm{~nm}$ yields the prediction that a distribution of $b$ produces variations in exciton energy of $\partial E_{\mathrm{X}}^{(1)} / \partial b=-88 \mathrm{meV} \mathrm{nm}^{-1}$ (decrease with increasing shell thickness) and variations in biexciton binding of $\partial E_{\mathrm{b}}^{(1)} / \partial b=26 \mathrm{meV} \mathrm{nm}^{-1}$ (increase with increasing shell thickness). The variations in the exciton energy $\partial E_{\mathrm{X}}^{(1)} / \partial b$ are mainly caused by variations in the confinement energy of the electron. Hence, our simple model explains the wider variations in single-QD exciton energy than in biexciton binding as well as the slightly negative correlations between the two parameters. The negative correlation can also explain the less repulsive biexciton on the single-QD level (on average $E_{\mathrm{b}}$ $=+4.0 \mathrm{meV}$ ) in comparison to the ensemble level (Figure 1ce, $\left.\bar{E}_{\mathrm{b}}=+14 \mathrm{meV}\right)$. Since both the biexciton efficiency ${ }^{26}$ and the biexciton shift (eq 6) increase with increasing shell thickness, we expect a biexciton-efficiency-weighted biexciton shift in ensemble measurements. This highlights the necessity of single-QD measurements to successfully characterize biexciton emission. Equations 3-6 can be used to predict and understand the inhomogeneous broadening of exciton and biexciton energies for other QD geometries.

To summarize, we have measured the biexciton shift and biexciton line width of single $\mathrm{CdSe} / \mathrm{CdS} / \mathrm{ZnS}$ QDs using cascade spectroscopy. We found that the line widths of biexciton and exciton emission are similar, although they vary between different single QDs. Variations in the biexciton shift are small in comparison to variations of the exciton energy, and the two parameters are slightly negatively correlated. We can rationalize these variations with simple quantum-mechanical calculations, which point to a distribution of $\mathrm{CdS}$ shell thicknesses as the dominant contributor to inhomogeneous broadening. Our method and findings are relevant for a fundamental understanding of multicarrier interactions in colloidal QDs. Moreover, they can serve as a guide for synthesis procedures of core-shell nanocrystals, which will particularly benefit their use in high-power applications. 


\section{ASSOCIATED CONTENT}

\section{(s) Supporting Information}

The Supporting Information is available free of charge at https://pubs.acs.org/doi/10.1021/acs.nanolett.1c01556.

Experimental methods, details of data processing and calibration procedures, details of quantum-mechanical calculations, a derivation of the energy-resolved $g^{(2)}$, and an overview of all single-QD experiments (PDF)

\section{AUTHOR INFORMATION}

\section{Corresponding Author}

Freddy T. Rabouw - Debye Institute, Utrecht University,

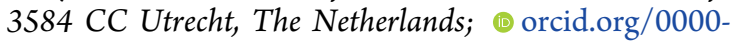
0002-4775-0859; Email: f.t.rabouw@uu.nl

\section{Authors}

Sander J. W. Vonk - Debye Institute, Utrecht University, 3584 CC Utrecht, The Netherlands; 1 orcid.org/0000-00024650-9473

Bart A. J. Heemskerk - Debye Institute, Utrecht University, 3584 CC Utrecht, The Netherlands

Robert C. Keitel - Optical Materials Engineering Laboratory, ETH Zurich, 8092 Zurich, Switzerland; ㅇo이.org/00000002-9412-8034

Stijn O. M. Hinterding - Debye Institute, Utrecht University, 3584 CC Utrecht, The Netherlands

Jaco J. Geuchies - Optoelectronic Materials Section, Faculty of Applied Sciences, Delft University of Technology, $2629 \mathrm{HZ}$ Delft, The Netherlands; 이이.0rid.org/0000-0002-0758-9140

Arjan J. Houtepen - Optoelectronic Materials Section, Faculty of Applied Sciences, Delft University of Technology, $2629 \mathrm{HZ}$ Delft, The Netherlands; 이이. orcid.org/0000-0001-8328443X

Complete contact information is available at:

https://pubs.acs.org/10.1021/acs.nanolett.1c01556

\section{Author Contributions}

The manuscript was written through contributions of all authors. All authors have given approval to the final version of the manuscript.

\section{Notes}

The authors declare no competing financial interest.

\section{ACKNOWLEDGMENTS}

This work was supported by the Dutch Research Council NWO (OCENW.KLEIN.008). F.T.R. acknowledges financial support from The Netherlands Organisation for Scientific Research NWO (VENI grant number 722.017.002) and The Netherlands Center for Multiscale Catalytic Energy Conversion (MCEC). J.J.G. and A.J.H. acknowledge support from European Research Council Horizon 2020 ERC Grant Agreement 678004 (Doping on Demand). R.C.K. acknowledges support from the European Research Council (ERC; FP/2007-2013), ERC Grant Agreement No. 339905 (QuaDoPS Advanced Grant). Peter Helfferich is thanked for technical support setting up the galvo-APD setup.

\section{REFERENCES}

(1) Murray, C. B.; Norris, D. J.; Bawendi, M. G. Synthesis and Characterization of Nearly Monodisperse CdE $(\mathrm{E}=\mathrm{S}$, Se, Te $)$ Semiconductor Nanocrystallites. J. Am. Chem. Soc. 1993, 115 (19), 8706-8715.
(2) Cui, J.; Beyler, A. P.; Marshall, L. F.; Chen, O.; Harris, D. K.; Wanger, D. D.; Brokmann, X.; Bawendi, M. G. Direct Probe of Spectral Inhomogeneity Reveals Synthetic Tunability of SingleNanocrystal Spectral Linewidths. Nat. Chem. 2013, 5 (7), 602-606.

(3) Cui, J.; Beyler, A. P.; Coropceanu, I.; Cleary, L.; Avila, T. R.; Chen, Y.; Cordero, J. M.; Heathcote, S. L.; Harris, D. K.; Chen, O.; et al. Evolution of the Single-Nanocrystal Photoluminescence Linewidth with Size and Shell: Implications for Exciton-Phonon Coupling and the Optimization of Spectral Linewidths. Nano Lett. 2016, 16 (1), 289-296.

(4) Utzat, H.; Shulenberger, K. E.; Achorn, O. B.; Nasilowski, M.; Sinclair, T. S.; Bawendi, M. G. Probing Linewidths and Biexciton Quantum Yields of Single Cesium Lead Halide Nanocrystals in Solution. Nano Lett. 2017, 17 (11), 6838-6846.

(5) Gellen, T. A.; Lem, J.; Turner, D. B. Probing Homogeneous Line Broadening in CdSe Nanocrystals Using Multidimensional Electronic Spectroscopy. Nano Lett. 2017, 17 (5), 2809-2815.

(6) Van Der Bok, J. C.; Dekker, D. M.; Peerlings, M. L. J.; Salzmann, B. B. V.; Meijerink, A. Luminescence Line Broadening of CdSe Nanoplatelets and Quantum Dots for Application in W-LEDs. J. Phys. Chem. C 2020, 124 (22), 12153-12160.

(7) Bae, W. K.; Park, Y. S.; Lim, J.; Lee, D.; Padilha, L. A.; McDaniel, H.; Robel, I.; Lee, C.; Pietryga, J. M.; Klimov, V. I. Controlling the Influence of Auger Recombination on the Performance of QuantumDot Light-Emitting Diodes. Nat. Commun. 2013, 4, 3661.

(8) Grim, J. Q.; Christodoulou, S.; Di Stasio, F.; Krahne, R.; Cingolani, R.; Manna, L.; Moreels, I. Continuous-Wave Biexciton Lasing at Room Temperature Using Solution-Processed Quantum Wells. Nat. Nanotechnol. 2014, 9 (11), 891-895.

(9) Hollingsworth, J. A. Heterostructuring Nanocrystal Quantum Dots toward Intentional Suppression of Blinking and Auger Recombination. Chem. Mater. 2013, 25 (8), 1318-1331.

(10) Pandey, A.; Guyot-Sionnest, P. Multicarrier Recombination in Colloidal Quantum Dots. J. Chem. Phys. 2007, 127 (11), 111104.

(11) He, Y. M.; Iff, O.; Lundt, N.; Baumann, V.; Davanco, M.; Srinivasan, K.; Höfling, S.; Schneider, C. Cascaded Emission of Single Photons from the Biexciton in Monolayered $\mathrm{WSe}_{2}$. Nat. Commun. 2016, 7, 13409.

(12) Huang, X.; Chen, L.; Zhang, C.; Qin, Z.; Yu, B.; Wang, X.; Xiao, M. Inhomogeneous Biexciton Binding in Perovskite Semiconductor Nanocrystals Measured with Two-Dimensional Spectroscopy. J. Phys. Chem. Lett. 2020, 11 (23), 10173-10181.

(13) Fernée, M. J.; Tamarat, P.; Lounis, B. Cryogenic SingleNanocrystal Spectroscopy: Reading the Spectral Fingerprint of Individual CdSe Quantum Dots. J. Phys. Chem. Lett. 2013, 4 (4), 609-618.

(14) Nair, G.; Zhao, J.; Bawendi, M. G. Biexciton Quantum Yield of Single Semiconductor Nanocrystals from Photon Statistics. Nano Lett. 2011, 11 (3), 1136-1140.

(15) Beyler, A. P.; Bischof, T. S.; Cui, J.; Coropceanu, I.; Harris, D. K.; Bawendi, M. G. Sample-Averaged Biexciton Quantum Yield Measured by Solution-Phase Photon Correlation. Nano Lett. 2014, 14 (12), 6792-6798.

(16) Amgar, D.; Yang, G.; Tenne, R.; Oron, D. Higher-Order Photon Correlation as a Tool to Study Exciton Dynamics in Quasi-2D Nanoplatelets. Nano Lett. 2019, 19 (12), 8741-8748.

(17) Fisher, B.; Caruge, J. M.; Zehnder, D.; Bawendi, M. RoomTemperature Ordered Photon Emission from Multiexciton States in Single CdSe Core-Shell Nanocrystals. Phys. Rev. Lett. 2005, 94 (8), No. 087403.

(18) Nasilowski, M.; Spinicelli, P.; Patriarche, G.; Dubertret, B. Gradient CdSe/CdS Quantum Dots with Room Temperature Biexciton Unity Quantum Yield. Nano Lett. 2015, 15 (6), 3953-3958.

(19) Bischof, T. S.; Correa, R. E.; Rosenberg, D.; Dauler, E. A.; Bawendi, M. G. Measurement of Emission Lifetime Dynamics and Biexciton Emission Quantum Yield of Individual InAs Colloidal Nanocrystals. Nano Lett. 2014, 14 (12), 6787-6791.

(20) Zhao, J.; Nair, G.; Fisher, B. R.; Bawendi, M. G. Challenge to the Charging Model of Semiconductor-Nanocrystal Fluorescence 
Intermittency from off-State Quantum Yields and Multiexciton Blinking. Phys. Rev. Lett. 2010, 104 (15), 157403.

(21) Shulenberger, K. E.; Bischof, T. S.; Caram, J. R.; Utzat, H.; Coropceanu, I.; Nienhaus, L.; Bawendi, M. G. Multiexciton Lifetimes Reveal Triexciton Emission Pathway in CdSe Nanocrystals. Nano Lett. 2018, 18 (8), 5153-5158.

(22) Park, Y. S.; Malko, A. V.; Vela, J.; Chen, Y.; Ghosh, Y.; GarcíaSantamaría, F.; Hollingsworth, J. A.; Klimov, V. I.; Htoon, H. NearUnity Quantum Yields of Biexciton Emission from CdSe/CdS Nanocrystals Measured Using Single-Particle Spectroscopy. Phys. Rev. Lett. 2011, 106 (18), 187401.

(23) Park, Y. S.; Bae, W. K.; Pietryga, J. M.; Klimov, V. I. Auger Recombination of Biexcitons and Negative and Positive Trions in Individual Quantum Dots. ACS Nano 2014, 8 (7), 7288-7296.

(24) Rabouw, F. T.; Lunnemann, P.; Van Dijk-Moes, R. J. A.; Frimmer, M.; Pietra, F.; Koenderink, A. F.; Vanmaekelbergh, D. Reduced Auger Recombination in Single CdSe/CdS Nanorods by One-Dimensional Electron Delocalization. Nano Lett. 2013, 13 (10), 4884-4892.

(25) Zhao, J.; Chen, O.; Strasfeld, D. B.; Bawendi, M. G. Biexciton Quantum Yield Heterogeneities in Single CdSe (CdS) Core (Shell) Nanocrystals and Its Correlation to Exciton Blinking. Nano Lett. 2012, 12 (9), 4477-4483.

(26) Rabouw, F. T.; Vaxenburg, R.; Bakulin, A. A.; van Dijk-Moes, R. J. A.; Bakker, H. J.; Rodina, A.; Lifshitz, E.; Efros, A. L.; Koenderink, A. F.; Vanmaekelbergh, D. Dynamics of Intraband and Interband Auger Processes in Colloidal Core-Shell Quantum Dots. ACS Nano 2015, 9 (10), 10366-10376.

(27) Klimov, V. I.; Ivanov, S. A.; Nanda, J.; Achermann, M.; Bezel, I.; McGuire, J. A.; Piryatinski, A. Single-Exciton Optical Gain in Semiconductor Nanocrystals. Nature 2007, 447 (7143), 441-446.

(28) Chen, O.; Zhao, J.; Chauhan, V. P.; Cui, J.; Wong, C.; Harris, D. K.; Wei, H.; Han, H. S.; Fukumura, D.; Jain, R. K.; et al. Compact High-Quality CdSe-CdS Core-Shell Nanocrystals with Narrow Emission Linewidths and Suppressed Blinking. Nat. Mater. 2013, 12 (5), 445-451.

(29) Xing, G.; Mathews, N.; Lim, S. S.; Yantara, N.; Liu, X.; Sabba, D.; Grätzel, M.; Mhaisalkar, S.; Sum, T. C. Low-Temperature Solution-Processed Wavelength-Tunable Perovskites for Lasing. Nat. Mater. 2014, 13 (5), 476-480.

(30) Bisschop, S.; Geiregat, P.; Aubert, T.; Hens, Z. The Impact of Core/Shell Sizes on the Optical Gain Characteristics of CdSe/CdS Quantum Dots. ACS Nano 2018, 12 (9), 9011-9021.

(31) Rabouw, F. T.; Kamp, M.; Van Dijk-Moes, R. J. A.; Gamelin, D. R.; Koenderink, A. F.; Meijerink, A.; Vanmaekelbergh, D. Delayed Exciton Emission and Its Relation to Blinking in CdSe Quantum Dots. Nano Lett. 2015, 15 (11), 7718-7725.

(32) Akselrod, G. M.; Prins, F.; Poulikakos, L. V.; Lee, E. M. Y.; Weidman, M. C.; Mork, A. J.; Willard, A. P.; Bulović, V.; Tisdale, W. A. Subdiffusive Exciton Transport in Quantum Dot Solids. Nano Lett. 2014, 14 (6), 3556-3562.

(33) Deutsch, Z.; Schwartz, O.; Tenne, R.; Popovitz-Biro, R.; Oron, D. Two-Color Antibunching from Band-Gap Engineered Colloidal Semiconductor Nanocrystals. Nano Lett. 2012, 12 (6), 2948-2952.

(34) Gindele, F.; Hild, K.; Langbein, W.; Woggon, U. TemperatureDependent Line Widths of Single Excitons and Biexcitons. J. Lumin. 2000, 87, 381-383.

(35) Gindele, F.; Hild, K.; Langbein, W.; Woggon, U. Phonon Interaction of Single Excitons and Biexcitons. Phys. Rev. B 1999, 60 (4), R2157-R2160.

(36) Korkusinski, M.; Voznyy, O.; Hawrylak, P. Fine Structure and Size Dependence of Exciton and Biexciton Optical Spectra in CdSe Nanocrystals. Phys. Rev. B: Condens. Matter Mater. Phys. 2010, 82 (24), 245304.

(37) Hanifi, D. A.; Bronstein, N. D.; Koscher, B. A.; Nett, Z.; Swabeck, J. K.; Takano, K.; Schwartzberg, A. M.; Maserati, L.; et al. Redefining near-unity luminescence in quantum dots with photothermal quantum yield. Science 2019, 363 (6432), 1199-1202.
(38) Boldt, K.; Kirkwood, N.; Beane, G. A.; Mulvaney, P. Synthesis of Highly Luminescent and Photo-Stable, Graded Shell CdSe/ $\mathrm{Cd}_{x} \mathrm{Zn}_{1-x} \mathrm{~S}$ Nanoparticles by in Situ Alloying. Chem. Mater. 2013, 25 (23), 4731-4738.

(39) Prins, P. T.; Montanarella, F.; Dümbgen, K.; Justo, Y.; Van der Bok, J. C.; Hinterding, S. O. M.; Geuchies, J. J.; Maes, J.; De Nolf, K.; Deelen, S.; et al. Extended Nucleation and Superfocusing in Colloidal Semiconductor Nanocrystal Synthesis. Nano Lett. 2021, 21 (6), 2487-2496.

(40) Sung, Y.; Park, K.; Lee, Y.; Kim, T. Ripening Kinetics of CdSe/ ZnSe Core/Shell Nanocrystals. J. Phys. Chem. C 2007, 111 (3), 1239-1242. 